Musées, Patrimoine et Culture scientifiques et techniques

$153 \mid 2014$

mai-juin 2014

\title{
Une autre lecture : matériel de fouilles et collections patrimoniales au musée national de Préhistoire des Eyzies-de-Tayac en Dordogne
}

\section{Antoine Chancerel}

\section{OpenEdition}

Journals

Édition électronique

URL : http://journals.openedition.org/ocim/1399

DOI : 10.4000/ocim.1399

ISSN : 2108-646X

Éditeur

OCIM

Édition imprimée

Date de publication : 25 juin 2014

ISSN : 0994-1908

Référence électronique

Antoine Chancerel, « Une autre lecture : matériel de fouilles et collections patrimoniales au musée national de Préhistoire des Eyzies-de-Tayac en Dordogne ", La Lettre de I'OCIM [En ligne], 153 | 2014, mis en ligne le 25 juin 2016, consulté le 19 avril 2019. URL : http://journals.openedition.org/ocim/1399 ; DOI : 10.4000/ocim.1399 


\title{
Une autre lecture : matériel de fouilles et collections patrimoniales au musée national de Préhistoire des Eyzies-de-Tayac en Dordogne
}

\author{
Antoine Chancerel \\ Conservateur en chef du patrimoine, \\ en charge du récolement décennal des collections \\ et du département de Préhistoire récente
}

La note-circulaire du 19 juillet 2012 sur les «matériels d'étude » fait la part belle aux objets archéologiques issus de fouilles programmées, d'opérations préventives, de prospections et de découvertes fortuites. Une lecture un peu rapide et superficielle du texte conduirait à l'idée que ces objets sont les biens par excellence sur lesquels pourraient s'appliquer les dispositions énoncées (cf. le 1.1 de la note, www.culture.gouv. $\mathrm{fr} /$ documentation/joconde/fr/partenaires/AIDEMUSEES/materiels-etude.pdf) et que leur gestion peut aisément s'accommoder en bout de course d'un classement en dehors du champ des biens inaliénables et imprescriptibles. Il n'en est évidemment rien et tel n'a pas été l'esprit de ses concepteurs. Il reste cependant que le nombre de fois où ces objets archéologiques sont mentionnés dans le texte ne laisse pas de doute sur le caractère problématique qu'ils peuvent avoir pour bien des gestionnaires de collections de musées. Cette récurrence traduit également en creux le fait qu'ils constituent un domaine à part dont la spécificité impose une prise en compte distincte du lot habituel des fonds des musées.

Le musée national de Préhistoire a fait de ces objets archéologiques l'essence même de sa collection. Dans leur immense majorité, ils ont été patrimonialisés et, de ce fait, inscrits à l'inventaire. La question de leur intégration au domaine public, ici de l'État, s'est toujours imposée comme une évidence pour les quatre directeurs qui se sont succédés à la tête de l'établissement depuis sa création en 1923. Les objets archéologiques sont en effet les témoins des occupations humaines du passé, et parfois, comme en Préhistoire, les seuls disponibles pour l'étude et la présentation. Le principe même de leur conservation n'a donc jamais fait débat. Ces objets sont en effet rares et leur acquisition a souvent nécessité des protocoles de fouilles patients et coûteux. Ils sont uniques et, même dans le cas de productions standardisées (comme certaines céramiques antiques), le gisement qui les contient avec

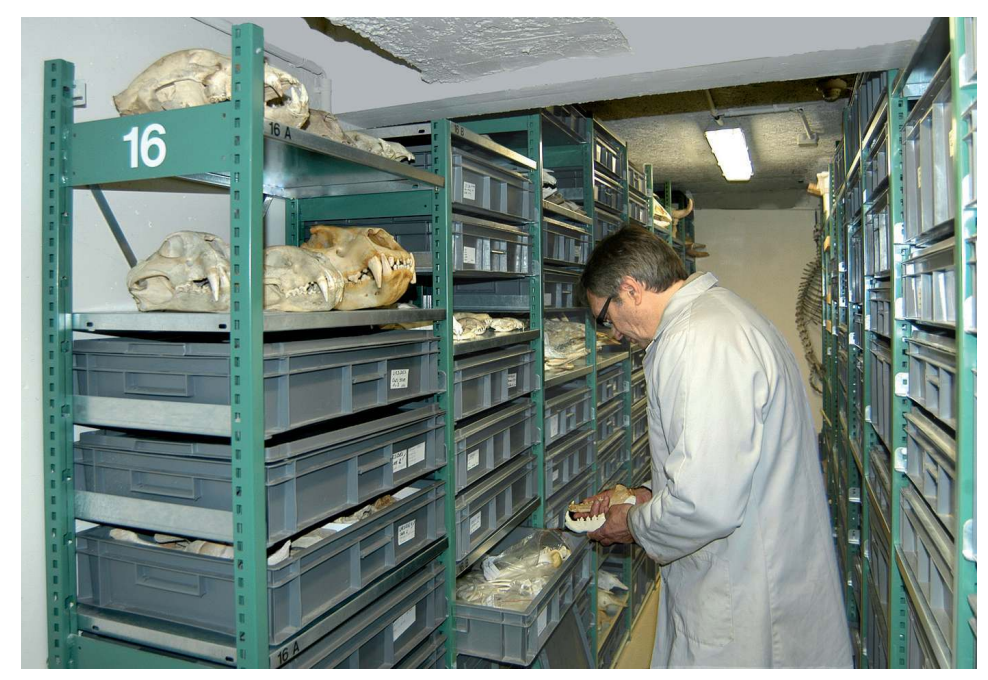

La collection de comparaison archéozoologique (c) MNP/P. Jugie

sa somme de relations inter-objets est unique. Ils sont de plus non reproductibles. Contrairement, par exemple, à l'agronome étudiant des salades et qui peut toujours en replanter, l'archéologue n'a pas d'autre choix pour progresser que de consommer toujours un peu plus une ressource archéologique finie. Ces qualités du patrimoine archéologique sont essentielles pour comprendre leur valeur insigne, car après la fouille, ce qui va rester d'un gisement, et qui permettra d'en reprendre éventuellement l'étude à l'aune de nouvelles problématiques, sera la collection d'objets, assortie le cas échéant de la documentation afférente. Nombre de gisements fouillés ne sont plus accessibles aujourd'hui qu'au travers des vestiges qui y ont été recueillis. Il importe donc d'en assurer la préservation avec toute l'énergie possible.

Fort de son exceptionnelle collection (6,5 millions d'objets issus des plus grands gisements paléolithiques français), le musée des Eyzies accueille ainsi en permanence des chercheurs venus du monde entier. Et comme 
par un fait exprès, les collections les plus sollicitées sont celles des gisements classiques fouillés au début du siècle et dans les années 1960. La recherche archéologique a en effet cette singularité de s'appuyer sur la totalité des découvertes déjà faites, qưelles soient récentes ou très anciennes : tel vase à décor rare reste signifiant même s'il a été exhumé il y a un siècle.

Le récolement en cours a été l'occasion de formaliser un certain nombre de principes de gestion à valeur plus générale. Le premier, tout à fait fondamental, est que les collections archéologiques sont, par essence, sérielles. Les objets n'ont de sens (et de raison d'être gardés) qu'associés à un contexte de découverte et au sein d'un ensemble pertinent. Cette qualité spécifique en conditionne le mode de gestion par lots, que ceux-ci comportent des milliers de pièces ou une seule. Le second est que la clé d'entrée de tout le système de référencement des objets est le gisement d'où ils proviennent. Cette pratique de bon sens, et seule cohérente avec la législation archéologique, se satisfait cependant difficilement des applications informatiques muséales actuelles.

Des fonds d'étude hors-inventaire sont également présents à titre d'ensembles de comparaison : série d'ossements actuels pour les déterminations des faunes

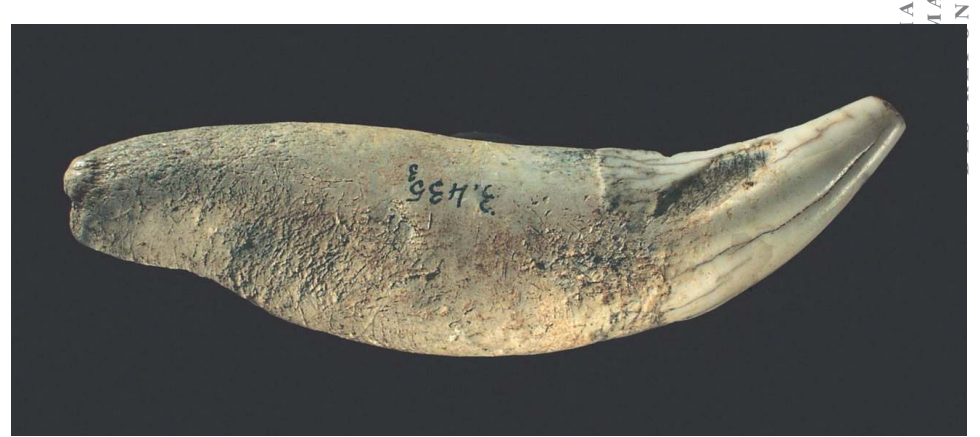

Canine d'ours trouvée lors des fouilles de 1902 à 1930 sur I'habitat néandertalien de la Ferrassie (Dordogne). Les traces superficielles d'impact, passées inaperçues à l'époque, ont pu être interprétées récemment grâce à des expérimentations. Eles résultent d'un usage en percuteur tendre pour la retouche des racloirs en silex. ๑ $R M N / P$. Jugie MNP

archéologiques, lithothèque pour celles des roches employées à la confection d'outils préhistoriques, résultats d'expérimentations, fonds ethnographiques, moulages... Â la marge, ils peuvent aussi concerner des séries ayant perdu leur contexte de découverte ou mélangées (comme par exemple le fonds Hauser entièrement sinistré lors de la première guerre mondiale) et qui, à défaut d'intérêt scientifique, ont acquis malgré tout avec le temps un intérêt historique. 